\title{
A HISTOCHEMICAL INVESTIGATION OF THE LEUCOCYTES IN KWASHIORKOR*
}

The present investigation was carried out in conjunction with a series of biochemical and haematological studies of the blood in kwashiorkor conducted at the Pretoria General Hospital (Kinnear and Pretorius, 1956 and 1957) in order to extend the overall picture of the state of various tissues in this malnutrition syndrome. For a complete review of the application of histochemical procedures to leucocytes the reader is referred to Wachstein (1955).

\section{Material and Methods}

In the present investigation histochemical tests for lipids, ribonucleic acid (RNA), desoxyribonucleic acid (DNA), glycogen, mucoproteins, peroxidase, and alkaline phosphatase were carried out.

The tests were performed on smears prepared from samples of peripheral blood taken from each of 20 Bantu infants suffering from kwashiorkor. The tests were carried out at the beginning of treatment and at weekly intervals thereafter. The average number of smears studied per patient was five, but in some cases smears were obtained over a period of 11 weeks. In all, about 500 separate histochemical tests were made.

Blood smears taken from 10 healthy Bantu children were used as controls and, to afford a further comparison, smears from eight adult whites were also examined.

The "scoring" method of Kaplow (1955) was adopted in order to enable the intracellular alkaline phosphatase activities of blood smears from different individuals to be evaluated and compared. This method involves the grading of 100 successive leucocytes according to colour intensity to provide an index of phosphatase activity. The range of scores employed is from 100 to 400 . Kaplow was able to demonstrate alkaline phosphatase activity only in neutrophils, and scoring for alkaline phosphatase activity was therefore confined to neutrophils.

The same scoring method was also applied to evaluate lipid content and peroxidase activity, granulocytes being tested in these cases and the degree of granulation being recorded instead of

*This paper is published with the permission of the South African Council for Scientific and Industrial Research, Pretoria. colour intensity. Indications of fat or peroxidase in monocytes were disregarded. In the case of $\vec{N}$ glycogen, RNA, DNA, and mucoproteins, only on qualitative judgments were made of all types of on leucocytes.

The shortcomings of subjective quantitation with 을 histochemical methods are obvious, and the applica- tion of such methods is open to criticism. In order $\mathbb{P}$ to reduce the errors as much as possible, strict attention was paid to standardization. The time of treatment of the preparations, the age of the reagents, and the temperature at which they were kept were $\mathbb{D}$ maintained as uniform as possible. The stain for $\vec{\theta}$ lipids (Sudan black B) was not used if older thab of three days. All observations from the slides we made by one author only.

It was considered advisable to confine scoring to portion of each slide where the thickness of the smear was even; this entailed disregarding the "tail" of the smear. Readings were taken along rows on the slide, according to the "strip" method.

The following techniques were used:

Lipids.-Sudan black $B$ and May-GrünwaldGiemsa technique (Baillif and Kimbrough, 1947).

Peroxidase.-Copper sulphate method of Sato and Sekiya, as described by Bessis (1956), using safranine as counterstain.

DNA.-Feulgen reaction, as described by Darmady and Davenport (1954).

RNA.-Unna-Pappenheim stain, as described by White (1947). Control smears were treated with ribonuclease in veronal acetate buffer at $p \mathrm{H} 6.8$.

Glycogen. - Periodic-acid-Schiff method, as $\frac{D}{0}$ described by Hotchkiss (1948), with light green as counterstain. Control smears were treated with o saliva.

Mucoproteins.-Toluidine blue method of Lison, as $N$ described by Bessis (1956).

Alkaline Phosphatase.-Method of Gomori (1941), ত using as counterstain neutral red.

\section{Results}

Lipids.-The results obtained fell into two 0 distinct classes. In the case of the first, which ${ }^{\circ}$ applied to half of the patients studied, the fat 
content of the granulocytes was medium initially (score 202), rose to a peak in the second week, and then declined until the fifth week (Figs. 1, 2, 3, Table I). In the case of the remainder of the patients, there was a high initial fat content (score 243) which fell in the second week. A second peak, lower than the initial one, occurred in the third week. There was then a decline, followed by yet another rise (Fig. 3, Table I).

TABLE I

HISTOCHEMICAL "SCORES" FOR LIPID CONTENT AND ALKALINE PHOSPHATASE AND PEROXIDASE ACTIVITY OF LEUCOCYTES IN KWASHIORKOR PATIENTS AND IN NORMAL CONTROLS

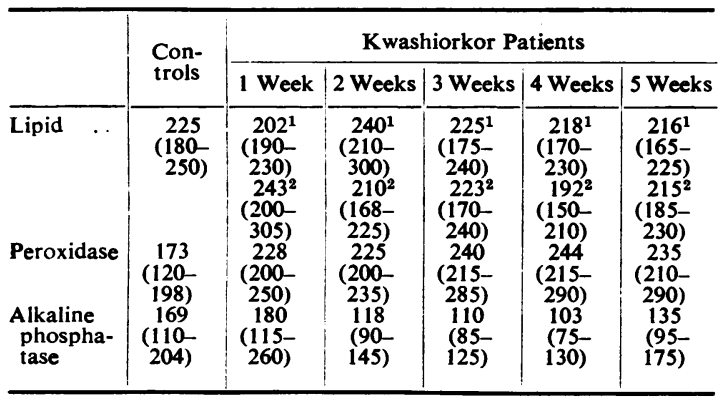

1 Group with initial lipid rise.

2 Group with initial lipid decline.

When the data shown in Figs. 2 and 3 are combined (Fig. 1) it is seen that the average fat content for all patients is fairly constant over a period of five weeks, with a low peak in the second week and a slight subsequent decline. The average score in the first week was 223 . In the

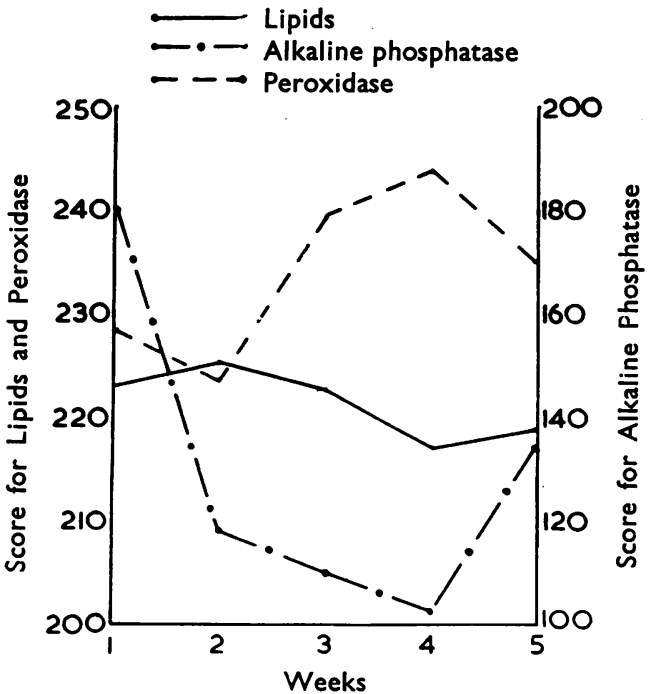

FIG. 1.-Average lipid, peroxidase, and alkaline phosphatase curves of all the patients.

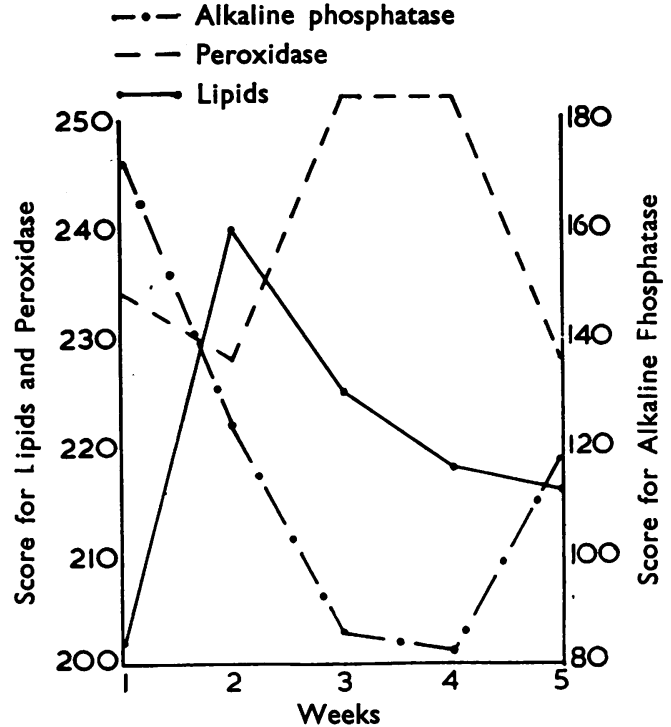

FIG. 2.-Average lipid, peroxidase, and alkaline phosphatase curves of group with initial lipid rise.

controls the granulocyte fat content was either medium or high, both in the case of adult whites and of Bantu children, and remained remarkably constant over a period of five weeks. The average score for the children was 225 .
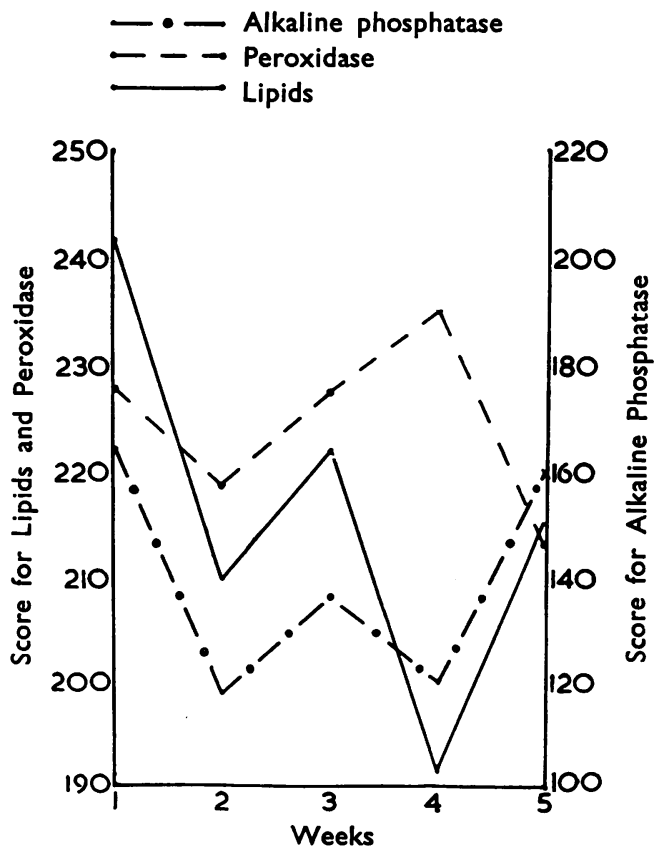

FIG. 3.-Average lipid, peroxidase, and alkaline phosphatase curves of group with initial lipid decline. 


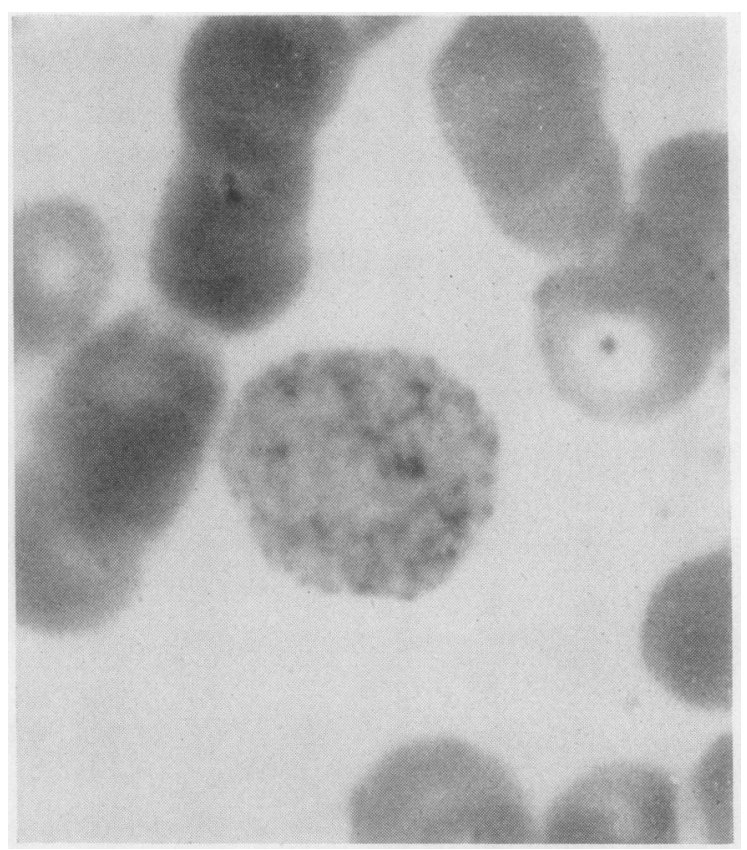

FIG. 4.-Lipid staining of neutrophil of kwashiorkor patient at beginning of treatment. Sudan black B, $\times 800$.

Kinnear and Pretorius (1957) found that in kwashiorkor the total serum lipids are low initially, rise to a peak in a week and then decline. The latter biochemical findings show some analogy to the white cell lipid data of the first type, but not to those of the second type.

Three successive liver biopsies were carried out at intervals of 14 days on each of 12 patients with kwashiorkor, in order to determine the degree of fatty infiltration of the liver. At the second biopsy it was obvious that the fat content of the liver had significantly decreased. The decrease in liver fat content occurs at the same time as the increase in total serum lipids, and is in conformity with the histochemical lipid results of the first type but not with those of the second type.

In order to see if any relation existed between the lipid content and the "age" of granulocytes, Cooke counts were made on smears taken at random during the investigation and stained with Wright's stain. Shifts both to the left and to the right were found to occur at different times, but, in general, more fat was present in "older" than in "younger" cells.

Peroxidase.-Fig. 1 shows that the peroxidase and lipid values are more or less inversely related. This is also roughly the case in Fig. 2. From Fig. 3 it can be seen that initially the curvès for

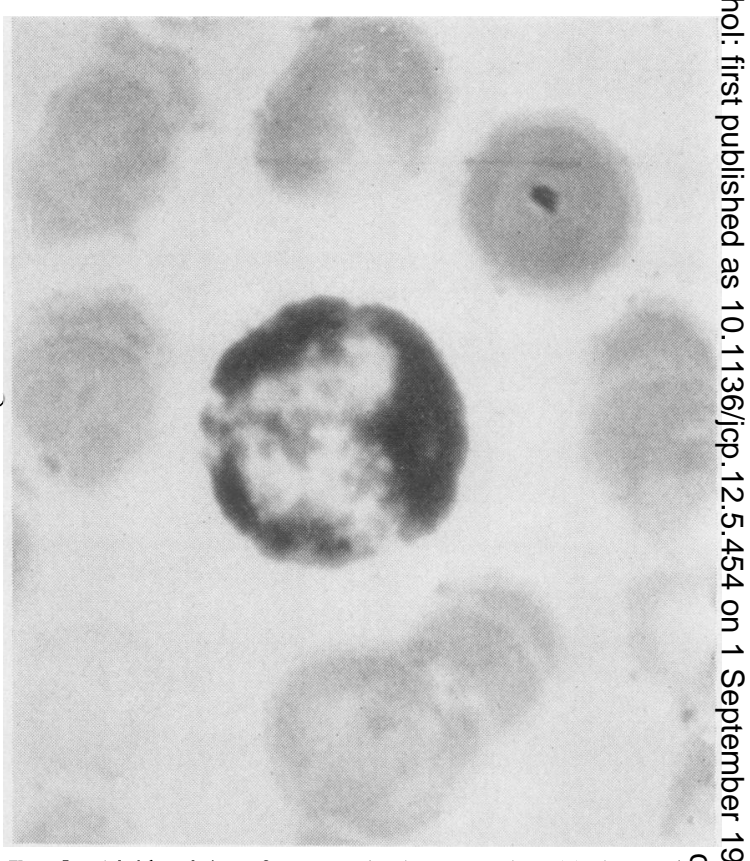

FIG. 5.-Lipid staining of neutrophil of the same kwashiorkor patiest as in Fig. 4 after 14 days' treatment. Sudan black B, $\times 800.0$

fat and peroxidase tend to run parallel, but that the relationship later becomes roughly inverse. In all three figures, the peroxidase values show a high peak in the third and fourth weeks.

In the controls the peroxidase activity was much lower than in the kwashiorkor patients. The average score and range in controls was 173 $(120-198)$ and in kwashiorkor patients 234 (177-310).

Glycogen.-In the majority of the granulocytes glycogen was demonstrated as a diffuse pink coloration in the cytoplasm. A smaller number of granulocytes showed spots throughout the cytoplasm, representing concentrated deposits. In monocytes and lymphocytes, glycogen was dispersed throughout the cytoplasm in granular form.

No differences in colour intensity were observed when comparisons were made between smears at different stages of treatment in the same patient, or between smears obtained from different patients. A comparison between smears from patients and controls also showed no differences.

Nucleic Acids.-Both with DNA and RNA, no differences could be detected between smears taken at different stages of treatment, or between smears from patients and controls. 
Mucoproteins.-As in the case of glycogen and nucleic acids, no detectable differences were found at different stages during the disease, or between the intensity of coloration in the nuclei of leucocytes from kwashiorkor patients and controls.

Alkaline Phosphatase.-The activity was found to be fairly high at the start of treatment, with an average score of about 180 (Fig. 1, Table I). During treatment, the level of phosphatase activity dropped sharply, although not to a level which could be considered as low, and then rose again in the fifth week.

For the group of control Bantu children the average score wsa 169 as compared with 130 for the kwashiorkor patients. In the case of the white adults the average score was 48 .

\section{Discussion}

Although definite figures are given in the case of the results for lipid content and peroxidase and alkaline phosphatase activity, it must be emphasized again that the methods used were of a semi-quantitative nature. No definite conclusions can therefore be reached on the basis of these results alone and certain apparent trends can merely be pointed out.

For the same reason the significance of the findings is difficult to assess at the moment. The conclusions of Wachstein (1955) are, however, of interest. In connexion with the results for lipids he states that significant changes in the lipid staining of leucocytes have not been found under pathological conditions, except in chronic myeloid leukaemia and infectious diseases, where a small proportion may not contain fat. He mentions the fact that sudanophilic granules in myeloid cells increase in number with increasing maturity of the cell.

In the present work, when both types of lipid results are grouped together (Fig. 1), it is seen that the granulocytic fat content remains more or less constant during treatment. There was a slight rise in the second week, when there was a concomitant decrease in the fat content of the liver as revealed by liver biopsies. It therefore seems possible that the granulocyte may serve as a vehicle for the transport of lipids released from the liver during the treatment of kwashiorkor. This possibility obviously requires further investigation, and it is proposed to perform chemical fractionation studies of the lipids of the liver and the leucocytes at different stages during the treatment of kwashiorkor patients.
The fact that in a large number of cases the fat content was high initially, declined, and later rose, seems to indicate that individual factors come into play at different times for reasons which are at present obscure. A factor which may be mentioned is the state of viability of granulocytes, which was revealed by Cooke counts to be subject to temporary variations.

The higher peroxidase activity of the leucocytes in kwashiorkor patients as compared with normal controls is difficult to evaluate. Seabra (1955) found that the peroxidase activity in leucocytes decreased under stress conditions, but high peroxidase activity was noted without exception in all the kwashiorkor patients. Furthermore, the activity showed a tendency to rise still further during treatment and reached a peak in the third to the fourth week.

No detectable differences in staining intensity were found with respect to glycogen, nucleic acids, or mucoproteins. Possibly, with microspectrophotometric methods, some changes might be detected. On the basis of microchemical analyses of liver tissue from infants with manifestations of kwashiorkor, Waterlow (1955) concluded that the RNA in liver is low in relation to DNA and rises during treatment. No difference of this kind was noted in the leucocytes. Waterlow and Weisz (1956) found no change in the glycogen content of the liver in kwashiorkor, an observation which agrees with our histochemical findings on leucocytes.

The fact that the alkaline phosphatase values declined during treatment suggests some degree of similarity between the present results and those of Schwartz (1956) for serum alkaline phosphatase. Certain points of difference, however, must be noted. Schwartz found that serum alkaline phosphatase in kwashiorkor was low at the beginning of treatment, dropped still lower during the first week or two of treatment, and then gradually rose to the level considered normal. In the present work, the initial activity in granulocytes was seen to be fairly close to the average obtained for normal children. It fell, though not to exceptionally low levels, during treatment, to rise once more rather abruptly in the fifth week to almost " normal" levels. It is in the form of the curve, rather than in the degree of phosphatase activity, that some resemblance is seen to the serum results.

Kaplow (1955) and Wiltshaw and Moloney (1955), the latter authors working on separated leucocytes, investigated leucocyte alkaline phosphatase activity in health and disease and 
found no relationship between serum alkaline phosphatase and leucocyte alkaline phosphatase activity. Wiltshaw and Moloney concluded that the cellular alkaline phosphatase activity was not regulated in vitro by any factor in the serum, but was intrinsic to the leucocyte itself. It is obvious that, before our results for leucocyte alkaline phosphatase activity in kwashiorkor can be evaluated, further studies in vivo similar to the above studies of Wiltshaw and Moloney in vitro will have to be undertaken.

Summary
Histochemical tests for lipids, glycogen,
mucoproteins, alkaline phosphatase, and
peroxidase were carried out on blood smears
from 20 patients with kwashiorkor and 10
control subjects. In half of the patients studied
the lipid content of the leucocytes tended to rise
during the first two weeks of treatment, with a
slight subsequent decline. In the remainder of the
patients there was a drop in the lipid content in
the first two weeks. The peroxidase activity of
the leucocytes was higher in kwashiorkor patients

than in normal controls. With respect to glycogen, mucoproteins, or nucleic acids, no differences were detected by the methods used, either in the same patient at different stages of treatment or between patients and controls. Alkaline phosphatase activity was found to be fairly high at the beginning of treatment, to fall during treatment, and to rise again in the fifth week of treatment. The possible significance of the findings is discussed.

\section{REFERENCES}

Baillif, R. N., and Kimbrough, C. (1947). J. Lab. clin. Med., 32, 155. Bessis, M. (1956). Cytology of the Blood and Blood-forming Organs. Grune and Stratton, New York.

Darmady, E. M., and Davenport, S. G. T. (1954). Haematological Technique. Churchill, London.

Gomori, G. (1941). J. cell. comp. Physiol., 17, 71.

Hotchkiss, R. D. (1948). Arch. Biochem., 16, 131.

Kaplow, L. S. (1955). Blood, 10, 1023.

Kinnear, A. A., and Pretorius, P. J. (1956). Brit. med. J., 1, 1528.

- (1957). S. Afr. med. J., 31, 174.

Schwartz, R. (1956). J. clin. Path., 9, 333.

Seabra, P. (1955). Ann. N.Y. Acad. Sci., 59, 1022.

Wachstein, M. (1955). Ibid., 59, 1052.

Waterlow, J. C. (1955). Protein Malnutrition. Proceedings of a Conference in Jamaica, 1953, ed. J. C. Waterlow. Cambridge Univ. Press, London.

- and Weisz, T. (1956). J. clin. Invest., 35, 346.

White, J. C. (1947). J. Path. Bact., 59, 223.

Wiltshaw, E., and Moloney, W. C. (1955). Blood, 10, 1120. 